IFT-05-05, KAIST-TH 2005/04, DESY-05-052

\title{
Soft Supersymmetry Breaking in KKLT Flux Compactification
}

\author{
K. Choi ${ }^{a}$, A. Falkowski ${ }^{b c}$, H. P. Nilles ${ }^{d e}$, M. Olechowski ${ }^{b}$ \\ ${ }^{a}$ Department of Physics, Korea Advanced Institute of Science and Technology \\ Daejeon 305-701, Korea \\ ${ }^{b}$ Institute of Theoretical Physics, Warsaw University \\ ul. Hoża 69, PL-00 681 Warsaw, Poland \\ ${ }^{c}$ Deutsches Elektronen-Synchrotron DESY \\ Notkestrasse 85, 22607 Hamburg, Germany \\ ${ }^{d}$ Physikalisches Institut, Universität Bonn \\ Nussallee 12, D-53115 Bonn, Germany \\ e Theory Division, Physics Department \\ CERN, CH-1211 Geneva 23, Switzerland
}

\begin{abstract}
We examine the structure of soft supersymmetry breaking terms in KKLT models of flux compactification with low energy supersymmetry. Moduli are stabilized by fluxes and nonperturbative dynamics while a de Sitter vacuum is obtained by adding supersymmetry breaking anti-branes. We discuss the characteristic pattern of mass scales in such a set-up as well as some features of $4 \mathrm{D} N=1$ supergravity breakdown by anti-branes. Anomaly mediation is found to always give an important contribution and one can easily arrange for flavor-independent soft terms. In its most attractive realization, the modulus mediation is comparable to the anomaly mediation, yielding a quite distinctive sparticle spectrum. In addition, the axion component of the modulus/dilaton superfield dynamically cancels the relative CP phase between the contributions of anomaly and modulus mediation, thereby avoiding dangerous SUSY CP violation.
\end{abstract}




\section{Introduction}

In this paper, we discuss soft terms in the KKLT scenario [1] with its three steps to achieve a supersymmetry breaking Minkowski (or de Sitter) vacuum, while stabilizing all moduli. This is a continuation of our previous paper [2] which discussed the stability and soft terms in flux compactification. The KKLT set-up has been proposed specifically within Type IIB theory on a Calabi-Yau orientifold and we will focus on this scenario here, noting that it will be straightforward to apply our analysis to similar scenarios in other string theories [2]. The first step of the procedure is to introduce the NS and RR three form fluxes [3], $H_{3}$ and $F_{3}$, stabilizing [4] the dilaton $S$ and all complex structure moduli $Z^{\alpha}$. We shall assume the existence of a set of flux vacua for which the resulting imaginary self dual $G_{3}=H_{3}-i S F_{3}$ is aligned nearly in the direction of a primitive $(2,1)$-form, which allows for realization of low-scale supersymmetry. Such fluxes might fix $m_{S, Z}$ rather close to the string or Planck scale, while keeping the gravitino mass much lower than $m_{S, Z}$. Thus high scale gauge unification and weak scale supersymmetry can be realized simultaneously. In the second step, one introduces nonperturbative dynamics, e.g. gaugino condensation [5], to stabilize the Kähler modulus $T$ at an $N=1$ supersymmetric AdS vacuum with $m_{T}=\mathcal{O}\left(4 \pi^{2} m_{3 / 2}\right)$ and vacuum energy density $V_{F} \simeq-3 m_{3 / 2}^{2} M_{P l}^{2}$. The third step amounts to adding anti-D3 branes (D3) stabilized at the tip of a Klebanov-Strassler type throat [6] which has been produced by fluxes [7. Such $\bar{D} 3$ can provide a positive contribution to the potential which would allow the fine tuning of the total vacuum energy density to the desired positive but small value. It induces also a SUSY breaking vacuum shift which would result in the soft terms of visible sector fields.

In fact, the KKLT set up can be seen as a specific example of a more general scenario (see also [8]) in which (i) most of the moduli are stabilized by a high scale dynamics that leaves $N=1$ SUSY (approximately) unbroken, (ii) a few light moduli which were unfixed by the high scale dynamics are stabilized by nonperturbative effects leading to an $N=1$ SUSY AdS vacuum, (iii) this SUSY AdS vacuum is uplifted to a SUSY-breaking Minkowski (or de Sitter) vacuum by branes which break $N=1$ SUSY explicitly. Many of our results will be relevant for any of such scenarios.

The scheme, although quite general, leads to a specific pattern of mass scales, as summarized in equation (20). The mass scales in the low energy theory exhibit a moderate hierarchy characterized by the factor $\log \left(M_{P l} / m_{3 / 2}\right)$ 
that is numerically $\mathcal{O}\left(4 \pi^{2}\right)$. The $F$-terms of the light moduli (and, in consequence, also the soft terms in the visible sector) are suppressed by this factor with respect to the gravitino mass, while the moduli masses are enhanced (by the same factor). This leads to interesting consequences for the pattern of soft terms and the cosmological moduli problem. In particular, when this scheme is realized in its most attractive form, the soft terms are determined by a mixed modulus-anomaly mediation yielding a sparticle spectrum which can be clearly distinguished from those predicted by other mediation mechanisms.

The organization of this paper is as follows. In the next section, we briefly review the KKLT set up, mainly focusing on the pattern of mass scales in those models that allow for low energy (weak scale) supersymmetry together with a vanishing cosmological constant. In Section 3, we discuss soft terms in $4 \mathrm{D} N=1$ supergravity coupled to a SUSY breaking antibrane, including the loop-induced anomaly mediated contributions [9] which, in the models under consideration, turn out to be equally important as the classical moduli mediated contributions [10]. In Section 4, we examine a variety of $4 \mathrm{D}$ models which can be identified as possible low energy limits of KKLT-type compactification. We compute vevs of the auxiliary components of the moduli and chiral compensator fields and discuss the characteristic pattern of the resulting soft terms for visible matter located on D3 or D7 branes. Phenomenological aspects of the scheme concerning the SUSY flavor and CP problems and also the low energy sparticle spectrums will be briefly addressed. Section 5 contains a critical summary and outlook.

\section{Scales in flux compactification}

\subsection{Mass scales in type IIB string theory}

In Type IIB theory compactified on CY orientifold, the 4D Planck scale $M_{P l}$ and the $4 \mathrm{D}$ gauge coupling constant $g_{p}$ on Dp branes wrapping $(p-3)$-cycle are given by (see e.g. [11])

$$
\begin{aligned}
M_{P l}^{2} & =\frac{2 e^{-2 \phi}}{(2 \pi)^{7} \alpha^{\prime 4}} V_{C Y}=4 \pi M_{s t}^{2}\left(\frac{e^{-\phi}}{2 \pi}\right)^{2}\left(M_{s t} R\right)^{6}, \\
\frac{1}{g_{p}^{2}} & =\frac{e^{-\phi}}{(2 \pi)^{(p-2)}\left(\alpha^{\prime}\right)^{(p-3) / 2}} V_{(p-3)}=\left(\frac{e^{-\phi}}{2 \pi}\right)\left(M_{s t} R\right)^{(p-3)},
\end{aligned}
$$


where $e^{\phi}=g_{s t}$ and $\alpha^{\prime}=1 / M_{s t}^{2}$ denote the string coupling and the string tension, respectively. Here we use the approximation that the $(p-3)$-cycle volume is given by $V_{(p-3)} \simeq(2 \pi R)^{(p-3)}$ for the compactification radius $R$ defined by the CY volume $V_{C Y} \equiv(2 \pi R)^{6}$. For D7/D3 system [12], the 4D dilaton/modulus chiral superfields $S$ and $T$ are defined as

$$
S=\frac{e^{-\phi}}{2 \pi}+i c_{0}, \quad T=\frac{e^{-\phi}}{2 \pi}\left(M_{s t} R\right)^{4}+i c_{4},
$$

where $c_{0}$ and $c_{4}$ are the axions from the RR 0 -form and 4-form, respectively.

With the above definitions of $S$ and $T$, the D7/D3 gauge kinetic functions are given by

$$
f_{7}=T, \quad f_{3}=S .
$$

Then gaugino condensation in a hidden sector on D7(D3) generates the superpotential

$$
W_{n p} \sim e^{-8 \pi^{2} T / b_{0}} \quad\left(e^{-8 \pi^{2} S / b_{0}}\right),
$$

where $b_{0}$ is the beta function coefficient of the gauge group in which the condensation occurs $\left(b_{0}=N_{c}\right.$ for a pure SYM theory with $S U\left(N_{c}\right)$ gauge group). On the other hand, the Euclidean D3 instanton wrapping the 4cycle of $T$ generates

$$
W_{n p} \sim e^{-8 \pi^{2} T}
$$

thus the effect of the D3 instantons is similar to that of gaugino condensation on D7.

It is convenient to express the ratios $M_{P l} / M_{s t}$ and $M_{s t} / M_{K K}\left(M_{K K} \equiv\right.$ $1 / R$ is the compactificaton scale of the CY manifold) in compactified string theory in terms of $S$ and $T$ [13, 14]. For the Type IIB theory under consideration, one easily finds

$$
\begin{aligned}
\frac{M_{P l}}{M_{s t}} & =4 \pi(\operatorname{Re}(S))^{1 / 4}(\operatorname{Re}(T))^{3 / 4}, \\
\frac{M_{s t}}{M_{K K}} & =\left(\frac{\operatorname{Re}(T)}{\operatorname{Re}(S)}\right)^{1 / 4} .
\end{aligned}
$$

As we can read off from equation (6), the ratios are determined by the vacuum expectation value of the $T$ and $S$ moduli. In concrete mechanisms of moduli stabilization, a possible range of these vevs is usually constrained. We now investigate this issue in the set-up proposed by KKLT. 


\subsection{Mass scales in the KKLT scheme}

In the leading approximation, the Kähler potential of the closed string moduli, i.e. $S, T$ and the complex structure moduli $Z^{\alpha}$, is given by

$$
K=-\ln \left(S+S^{*}\right)-3 \ln \left(T+T^{*}\right)-\ln i \int \Omega \wedge \Omega^{*},
$$

where $\Omega$ is the holomorphic $(3,0)$-form of CY. The quantized NS and RR fluxes over 3-cycles,

$$
M_{s t}^{2} \int_{C_{3}} H_{3}=4 \pi^{2} n, \quad M_{s t}^{2} \int_{C_{3}} F_{3}=4 \pi^{2} m,
$$

give rise [15 to the superpotential ${ }^{1}$

$$
W_{\text {flux }}=M_{s t}^{2} \int G_{3} \wedge \Omega,
$$

where $G_{3}=F_{3}-i S H_{3}$. Although the fluxes generate warped geometry, the warping is not significant in most of the region of CY except for a small region containing the Klebanov-Strassler throat. Then the dynamics of bulk degrees of freedom such as the moduli are not affected significantly by the warping.

The resulting supergravity equations of motion for $S$ and $Z^{\alpha}, D_{Z} W_{\text {flux }}=$ $D_{S} W_{\text {flux }}=0$, generically fix $S$ and $Z^{\alpha}$ at a value of order unity. The resulting $G_{3}$ is imaginary self-dual on CY, $G_{3}=i^{*} G_{3}$, and can be decomposed into two components: $\left\langle G_{3}\right\rangle=G_{(0,3)}+G_{(2,1)}$, where $\left\langle W_{\text {flux }}\right\rangle=M_{\text {st }}^{2} \int G_{(0,3)} \wedge \Omega$. In order to have weak scale supersymmetry without invoking to a large compactification radius (which, as we explain later, is not available in the KKLT set up) one needs to choose a flux configuration yielding $G_{3}$ almost aligned to the $(2,1)$-direction:

$$
\frac{G_{(0,3)}}{G_{(2,1)}} \ll 1
$$

Then the resulting flux-induced gravitino mass is suppressed as

$$
\left(m_{3 / 2}\right)_{\text {flux }}=M_{P l} e^{K / 2} W_{\text {flux }}=\mathcal{O}\left(\frac{e^{\phi}}{M_{s t}^{2} R^{3}} \frac{G_{(0,3)}}{G_{(2,1)}}\right),
$$

\footnotetext{
${ }^{1}$ In this paper we use the convention that the Kähler potential $K$ and the superpotential $W$ are dimensionless. These correspond to $K / M_{P l}^{2}$ and $W / M_{P l}^{3}$ for the standard dimensionful Kähler potential and superpotential of 4D SUGRA.
} 
Still the masses of $Z^{\alpha}$ and $S$ might receive a contribution from the unsuppressed $G_{(2,1)}$, thus

$$
m_{Z} \sim M_{P l} e^{K / 2} \frac{\partial_{Z}^{2} W_{\text {flux }}}{\partial_{Z} \partial_{Z^{*}} K}=\mathcal{O}\left(\frac{e^{\phi}}{M_{s t}^{2} R^{3}}\right),
$$

and a similar expression holds for $m_{S}$ with $\partial_{Z}^{2} W_{\text {flux }} \rightarrow \partial_{Z} \partial_{S} W_{\text {flux }}$.

The condition (10) requires a fine tuning, where the fluxes quantized in units of the string scale conspire to give only a tiny supersymmetry breaking effect. A study of the landscape of flux vacua suggests that the number of flux vacua with small $G_{(0,3)} / G_{(2,1)}$ scales as [16] $N_{\text {vac }}\left(G_{(0,3)} / G_{(2,1)} \leq \epsilon\right) \sim \epsilon^{2} N_{T O T}$, where $N_{T O T}$ denotes the total number of flux vacua. Since $N_{T O T}$ can be argued to be as large as $10^{300}$ for a typical CY orientifold, there could still be a large number of flux vacua for which $G_{(0,3)} / G_{(2,1)} \sim 10^{-13}$ and hence $m_{3 / 2}$ is in a $\mathrm{TeV}$ range. Thus, although this might not be easy to achieve in a specific model, the KKLT set-up might be able to accomodate the weak scale SUSY by fine-tuning the flux configuration in an appropriate manner.

The 3-form fluxes do not generate a potential for the Kähler modulus $T$ which corresponds to the size of a 4 -cycle $C_{4}$. To fix $T$, one needs to introduce additional dynamics depending on the volume of $C_{4}$. As discussed earlier, gaugino condensation on D7 branes wrapping $C_{4}$ and/or the Euclidean D3 instantons wrapping $C_{4}$ induce a nonperturbative superpotential of the form

$$
W_{n p}=C e^{-a T},
$$

where $C$ depends on $Z^{\alpha}$ in general, while $a$ is a real positive constant of $\mathcal{O}\left(4 \pi^{2}\right)$ (in our normalization of $T$ ).

For the flux configuration with $G_{(0,3)} / G_{(2,1)} \sim 10^{-13}$ yielding $m_{Z, S}=$ $\mathcal{O}\left(M_{P l}\right)$ and $m_{3 / 2}=\mathcal{O}(1) \mathrm{TeV}$, the stabilization of $T$ and also the SUSY breaking can be described by an effective SUGRA theory which is obtained after integrating out $S$ and $Z^{\alpha}$. This is tacitly assumed in the original setup of KKLT [1]. It requires a certain decoupling between the heavy fields $Z, S$ on one side and the light modulus $T$ on the other. In some cases (e.g. when $\left.\partial_{Z} \partial_{S} W_{\text {flux }} \ll \partial_{Z}^{2} W_{\text {flux }}\right)$, such a decoupling might not be possible [2] and a second modulus (e.g. $S$ ) might be light and remain in the low-energy effective theory. We shall later give examples of both of these situations, but stick to the simplest case in the following discussion.

The effective $N=1$ SUGRA thus contains the $T$-modulus as well as the SM gauge and chiral matter fields originating from D7/D3 branes. Although 
the whole set-up includes also SUSY breaking $\bar{D} 3$ branes, the VEV of $T$ is mainly determined by the $N=1$ SUSY sector described by the following effective Kähler and superpotential:

$$
\begin{aligned}
& K_{0}=-3 \ln \left(T+T^{*}\right), \\
& W_{0}=w_{0}-C e^{-a T},
\end{aligned}
$$

where $w_{0}=\left\langle W_{\text {flux }}\right\rangle=\mathcal{O}\left(G_{(0,3)} / G_{(2,1)}\right)$ and $C=\mathcal{O}(1)$ are constants in the effective theory. This effective theory successfully stabilizes $T$ as it has a unique SUSY AdS vacuum yielding

$$
\begin{aligned}
\langle a \operatorname{Re} T\rangle & \simeq \ln \left(M_{s t} / m_{3 / 2}\right)=\mathcal{O}\left(4 \pi^{2}\right) \\
m_{3 / 2} & =\left\langle M_{P l} e^{K} W_{0}\right\rangle \simeq \frac{M_{P l} w_{0}}{(2 \operatorname{Re} T)^{3 / 2}} \\
\left\langle V_{N=1}\right\rangle & =-3 m_{3 / 2}^{2} M_{P l}^{2}
\end{aligned}
$$

where $V_{N=1}$ is the standard $N=1$ SUGRA potential for the Kähler and superpotential of (14). Note that in the KKLT set-up the large weak/string scale hierarchy results in the appearance of a moderately large parameter $a \operatorname{Re} T$. This parameter will enter the expressions for SUSY breaking order parameters, leading to a moderate hierararchy between various soft masses. Another observation is that, since $a=\mathcal{O}(1) \div \mathcal{O}\left(4 \pi^{2}\right)$, we also obtain $\operatorname{Re} T=\mathcal{O}\left(4 \pi^{2}\right) \div \mathcal{O}(1)$. Larger values of $\operatorname{Re} T$ are not available within this stabilization scheme. In consequence, the resulting values of $g_{s t}$ and $M_{s t} R$ are rather close to unity, and both $M_{s t}$ and $M_{K K}$ are not far away from the Planck scale.

As we have remarked, for a gaugino condensation from $S U\left(N_{c}\right)$ SYM theory, $a=8 \pi^{2} / N_{c}$ and thus $\ln \left(M_{s t} / m_{3 / 2}\right) \approx 8 \pi^{2} / N_{c} g_{7}^{2}$, where $g_{7}^{2}$ is the $4 \mathrm{D}$ gauge coupling on D7 at the compactification scale. If the SM gauge fields originate from D7, this relation amounts to $w_{0} \sim e^{-16 \pi^{2} / N_{c}}$, implying that in this case the flux fine tuning for a hierarchically small $w_{0} \sim G_{(0,3)} / G_{(2,1)}$ is necessary also to get the correct value of the SM gauge coupling, unless $N_{c}$ is unusually large as $\mathcal{O}\left(8 \pi^{2}\right)$.

To obtain a phenomenologically desirable de Sitter or Minkowski vacuum, KKLT proposed to introduce a $\bar{D} 3$ providing a positive potential energy. In the presence of 3-form fluxes the geometry is warped, which can be parameterized as 4 ]

$$
d s^{2}=e^{2 A(y)}(\operatorname{Re}(T))^{-3 / 2} g_{\mu \nu}^{E} d x^{\mu} d x^{\nu}+e^{-2 A(y)}(\operatorname{Re}(T))^{1 / 2} \tilde{g}_{m n} d y^{m} d y^{n},
$$


where $g_{\mu \nu}^{E}$ is the $4 \mathrm{D}$ Einstein frame metric and $\tilde{g}_{m n}$ is a CY metric normalized as $\int d^{6} y \sqrt{\tilde{g}}=M_{s t}^{-6}$. Here we have ignored the fluctuation of $S$ and $Z^{\alpha}$ in the $10 \mathrm{D}$ metric since $m_{Z, S}=\mathcal{O}\left(M_{s t}\right)$. As long as the flux density is not strong, the warp factor $e^{A(y)}$ would be of order unity over the most region of CY, however it can be exponentially small around the small region of KlebanovStrassler (KS) throat. It is then a plausible assumption that the SM lives on D7/D3 which are stabilized in the region where the warping is not significant. On the other hand, $\bar{D} 3$ favors to be stabilized at the tip of the KS throat, $y=y_{\overline{D 3}}$, where the warp factor is minimal and exponentially small [4]:

$$
e^{A\left(y_{\bar{D} 3}\right)}=e^{A_{\min }} \sim(\operatorname{Re}(T))^{1 / 4} e^{-2 \pi n / g_{s t} m},
$$

where $n=\frac{1}{4 \pi^{2}} M_{s t}^{2} \int_{C_{K S}} H_{3}$ and $m=\frac{1}{4 \pi^{2}} M_{s t}^{2} \int_{\tilde{C}_{K S}} F_{3}$ are integers for the NS and RR fluxes over the collapsing 3-cycle $C_{K S}$ and its dual $\tilde{C}_{K S}$ of the KS throat. Adding the $\bar{D} 3$ tension to the negative vacuum energy density $V_{N=1} \simeq-3 m_{3 / 2} M_{P l}^{2}$ induced by gaugino condensation, the total vacuum energy density would be given by $V_{T O T} \approx V_{N=1}+V_{\bar{D} 3}$. Given this we see that it might be possible to adjust the observed value of the cosmological constant by a careful fine tuning of the parameters. A simple calculation of the $\bar{D} 3$ tension in the $4 \mathrm{D}$ Einstein frame gives

$$
V_{\bar{D} 3} \sim \frac{\left(e^{A_{\min }} M_{P l}\right)^{4}}{[\operatorname{Re}(T)]^{3}} \sim \frac{\left(e^{-2 \pi n / g_{s t} m} M_{P l}\right)^{4}}{[\operatorname{Re}(T)]^{2}}
$$

Then the condition $V_{T O T} \simeq 0$ requires that

$$
e^{A_{\min }} \sim \sqrt{m_{3 / 2} / M_{P l}} .
$$

With this warping the physical cutoff scale on $\bar{D} 3$ will be of the order of the intermediate scale $e^{A_{\min }} M_{s t} \sim \sqrt{m_{3 / 2} M_{P l}}$ and the $\bar{D} 3$ moduli acquire masses of $\mathcal{O}\left(e^{A_{\text {min }}} / M_{s t}^{2} R^{3}\right)$, also close to the intermediate scale.

As we see, only a limited class of flux vacua can give weak scale SUSY together with vanishing cosmological constant: those with flux configurations with $G_{(0,3)} / G_{(2,1)} \sim 10^{-13}$ and the warping $e^{A_{\min }} \sim \sqrt{G_{(0,3)} / G_{(2,1)}} \sim 3 \times 10^{-7}$. Such two step fine tunings might be a generic feature of any realistic model which incorporates the weak scale Higgs mass and the small cosmological constant within the string landscape whose typical mass scale is not far below $M_{P l}$. 
Below we summarize all the mass scales in KKLT set-up which realizes weak scale SUSY together with a vanishing vacuum energy density through the effective SUGRA (14) and the uplifting potential (18):

$$
\begin{aligned}
M_{s t} & \sim 5 \times 10^{17} \mathrm{GeV} \\
1 / R & \sim 10^{17} \mathrm{GeV} \\
m_{Z, S} & \sim \frac{1}{M_{s t}^{2} R^{3}} \sim 10^{16} \mathrm{GeV} \\
\Lambda_{G C} & =M_{s t} e^{-\langle a T\rangle / 3} \sim 10^{13} \mathrm{GeV}, \\
M_{\bar{D} 3} & \sim e^{A_{\text {min }}} M_{s t} \sim 10^{11} \mathrm{GeV}, \\
m_{T} & \sim\langle a T\rangle m_{3 / 2} \sim 10^{5} \mathrm{GeV}, \\
m_{3 / 2} & \sim \frac{1}{M_{s t}^{2} R^{3}}\left(\frac{G_{(0,3)}}{G_{(2,1)}}\right) \sim 10^{4} \mathrm{GeV}, \\
m_{\text {soft }} & \sim M_{\text {weak }} \sim \frac{m_{3 / 2}}{\langle a T\rangle} \sim 10^{2} \div 10^{3} \mathrm{GeV} .
\end{aligned}
$$

Here $\Lambda_{G C}$ is the dynamical scale of D7 gaugino condensation, $M_{\bar{D} 3}$ is the redshifted cutoff scale on $\bar{D} 3$, The expressions for $m_{T}$ and $m_{\text {soft }}$ will be derived in Section 3. There we shall also consider examples with more than one light modulus.

\subsection{Comments on alternative schemes}

So far we have considered a scenario with a TeV scale gravitino mass. Besides weak scale supersymmetry the consequence of this approach is that both the string scale $M_{s t}$ and compactification scale $M_{K K}$ are close to the the Planck scale (so that the standard high scale gauge unification is possible). An alternative [17] is to consider the fundamental string scale in Type IIB theory at an intermediate value $M_{s t} \sim 10^{10} \mathrm{GeV}$. From eq. (6) , this would require a stabilization of $S$ and $T$ at values satisfying

$$
\operatorname{Re}(S) \operatorname{Re}(T)^{3} \sim 10^{30} .
$$

This is difficult within the known mechanisms of moduli stabilization (but see [18) and is certainly not possible within the KKLT stabilization scheme. But even if that could be achieved there is another apparent problem. For the SUGRA approximation to be valid one needs $M_{K K} \leq M_{s t}$, which by eq. (6) requires $\operatorname{Re}(T) \geq \operatorname{Re}(S)$. Then $g_{7}^{2}=\operatorname{Re}(T)^{-1} \leq 3 \times 10^{-8}$, which means 
that the SM cannot be embedded in the D7 sector. One might avoid this conclusion by assuming that a 4 cycle has a volume $V_{4} \sim M_{s t}^{-4}$, so $g_{7}^{2} \sim 1$, while its dual 2 cycle has a huge volume $V_{2} \sim 10^{15} M_{s t}^{-2}$.

Another scheme might involve placing the D3 and/or D7 branes at a warped throat [19] and explain the hierarchy between the weak scale and the Planck scale by a strong warping instead of weak scale supersymmetry. We shall not discuss these schemes here, as we are mainly concerned with the question of soft SUSY breaking terms.

One might also be interested in alternatives to the ad-hoc uplifting procedure of KKLT. In Ref. [20], it has been proposed to uplift the AdS vacuum to a dS or Minkowski vacuum by introducing gauge field flux on a D7 brane. Such two-form flux would induce a moduli-dependent FI term and thus a positive $D$-term potential. A virtue of this scenario would be that the uplifting can be described within the standard 4D $N=1$ SUGRA framework, thus the resulting SUSY breaking can be interpreted as a spontaneous breaking.

To examine the possibility of $D$-term uplifting, let us briefly review the $F$ and $D$-term scalar potentials in generic 4 D SUGRA [21]. The scalar potential in the Einstein frame is $V=V_{F}+V_{D}$ where

$$
\begin{aligned}
V_{F} & =M_{P l}^{4} e^{K}\left(K^{I \bar{J}} D_{I} W D_{\bar{J}} W^{*}-3|W|^{2}\right), \\
V_{D} & =\frac{1}{2} M_{P l}^{4} \operatorname{Re}\left(f_{a}\right) D^{a} D^{a}=\frac{1}{2 \operatorname{Re}\left(f_{a}\right)} M_{P l}^{4}\left(i \eta_{a}^{I} \partial_{I} K-3 i r_{a}\right)^{2} .
\end{aligned}
$$

Here $D_{I} W=\partial_{I} W+\partial_{I} K W$ is the Kähler covariant derivative of the superpotential. Furthermore, $\eta_{a}^{I}$ denotes the gauge transformation of chiral superfields under the gauge group factor $G_{a}, \delta_{a} \Phi^{I}=\eta_{a}^{I}(\Phi)$, while $r_{a}$ is determined by the transformation properties of the superpotential under $G_{a}$,

$$
\delta_{a} W=\eta_{a}^{I} \partial_{I} W=-3 r_{a} W .
$$

This formalism can accomodate both a field dependent FI term accompanying the Green-Schwarz mechanism (when $\eta^{S}=i \delta_{G S}$ and $\partial_{S} K=-(S+\bar{S})^{-1}$ ) as well as a field independent FI term occuring in the presence of gauged $R$-symmetry (for $r_{a}=$ const $\neq 0$ ). But from (23) for $W \neq 0$ one easily finds that the D-terms can be rewritten as

$$
D^{a}=\frac{i}{\operatorname{Re}\left(f_{a}\right)} \frac{1}{W} \eta_{a}^{I} D_{I} W
$$

Obviously, an arbitrary supersymmetric configuration with $\left\langle D_{I} W\right\rangle=0$, if allowed, would be a stationary solution of $V_{F}$. It is clear that if $V_{F}$ admits 
a supersymmetric AdS vacuum, i.e. a stable solution with $\left\langle D_{I} W\right\rangle=0$ but $\langle W\rangle \neq 0$, as in the KKLT case, this SUSY AdS solution remains a good vacuum solution of the complete potential $V=V_{F}+V_{D}$. So in this case uplifting with a D-term will not work. If the absolute minimum is at $\left\langle D_{I} W\right\rangle=0, V_{D}$ improves the stability of the SUSY AdS vacuum of $V_{F}$ without lifting the vacuum energy density. Thus, if one wishes to get a (SUSY breaking) dS or Minkowski vacuum within the standard 4D SUGRA, one needs to have a special form of $W$ which does not admit a SUSY AdS solution. It remains an open question whether a viable model of this kind can be derived from string theory (see [22] for a recent study).

\section{Soft terms}

\section{1 $\mathrm{N}=1$ supergravity coupled to anti-D3 brane}

An intriguing feature of the KKLT scenario is the final step to introduce $\bar{D} 3$ which uplifts the SUSY AdS vacuum to a SUSY breaking Minkowski (or de Sitter) vacuum. The $N=1$ SUSY preserved by the combined dynamics of flux and gaugino condensation is not respected on the worldvolume of $\bar{D} 3$, in particular there are no light open string degrees of freedom on $\bar{D} 3$ which would form a $N=1$ supermultiplet. In this subsection, we discuss the $4 \mathrm{D}$ effective action describing the effects of $\bar{D} 3$ on the low energy dynamics of the light moduli and the SM fields in KKLT-type flux compactification.

Our main problem is to find the low energy couplings between the $N=0$ sector on $\bar{D} 3$ and the $N=1$ sector propagating in the $10 \mathrm{D}$ bulk or the D7/D3 worldvolumes. A full description of the couplings between a supersymmetric bulk sector and a less supersymmetric brane sector usually requires an offshell formulation of the bulk supersymmetry. In our case, such couplings can be described most conveniently by spurion operators in $N=1$ superspace. Since D7/D3 branes do not intersect with $\bar{D} 3$, spurion operators on the worldvolume of $\bar{D} 3$ do not depend on the fields confined in D7/D3, but generically depend on the bulk fields as well as on the fields confined in $\bar{D} 3$. It is rather generic that all scalar fields on $\bar{D} 3$ get a mass of the order of $e^{A_{\min }} M_{s t} \sim 10^{10} \mathrm{GeV}$ by the flux, thus are heavy enough to be decoupled at $\mathrm{TeV}$ scale. There may exist some light fields with non-zero spin on $\bar{D} 3$ but those will not affect our analysis of the soft terms. This allows us to focus on the $\bar{D} 3$ spurion operators depending only on the light bulk moduli and 
4D SUGRA multiplet. As we will see, in such situation the dominant effects of $\bar{D} 3$ on the other parts of the theory can be described by a single spurion operator which corresponds to the $\bar{D} 3$ tension.

Let us consider the 10D action at energies below $M_{s t}$ of the following form:

$$
S_{10 D}=\int d^{10} x\left[\mathcal{L}_{I I B}+\delta^{2}\left(z-z_{D 7}\right) \mathcal{L}_{D 7}+\delta^{6}\left(y-y_{D 3}\right) \mathcal{L}_{D 3}+\delta^{6}\left(y-y_{\bar{D} 3}\right) \mathcal{L}_{\bar{D} 3}\right],
$$

where $\mathcal{L}_{I I B}$ is the $10 \mathrm{D}$ IIB SUGRA action, $\mathcal{L}_{D p}$ denotes the worldvolume action of Dp brane, and $z$ and $y$ correspond to the transverse coordinates of D7 and D3 branes, respectively. The 4D effective action of the light fields is of the form $S_{4 D}=S_{N=1}+S_{\bar{D} 3}$, where $S_{N=1}$ is the $N=1$ SUGRA action originating from $\mathcal{L}_{I I B}$ and $\mathcal{L}_{D 7 / D 3}$, while $S_{\bar{D} 3}$ is from the non-supersymmetric $\mathcal{L}_{\overline{D 3}}$. The $N=1$ supersymmetric part can be written in the standard superspace form:

$$
\begin{aligned}
S_{N=1}= & \int d^{4} x \sqrt{g^{C}}\left[\int d^{4} \theta C C^{*}\left(-3 \exp \left(-K_{\text {eff }} / 3\right)\right)\right. \\
& \left.+\left\{\int d^{2} \theta\left(\frac{1}{4} f_{a} W^{a \alpha} W_{\alpha}^{a}+C^{3} W_{e f f}\right)+\text { h.c. }\right\}\right],
\end{aligned}
$$

where $g_{\mu \nu}^{C}=\left(C C^{*}\right)^{-1} e^{K_{e f f} / 3} g_{\mu \nu}^{E}$ for the 4D Einstein-frame metric $g_{\mu \nu}^{E}$ and the chiral compensator superfield $C=C_{0}+\theta^{2} F^{C}$, and the Kähler and superpotential can be expanded as

$$
\begin{aligned}
-3 \exp \left[-K_{e f f} / 3\right] & =-3 \exp \left[-K_{0}\left(\Phi^{m}, \Phi^{m *}\right) / 3\right]+Y_{i}\left(\Phi^{m}, \Phi^{m *}\right) Q_{i}^{*} Q_{i} \\
W_{e f f} & =W_{0}\left(\Phi^{m}\right)+\frac{1}{6} \lambda_{i j k}\left(\Phi^{m}\right) Q_{i} Q_{j} Q_{l} \\
f_{a} & =f_{a}\left(\Phi^{m}\right)
\end{aligned}
$$

where $\Phi^{m}$ denote the gauge singlet light moduli and $Q_{i}$ stand for the gauge charged matter fields. In (26), we have ignored the 4D SUGRA multiplet other than the metric component. In this scheme, $C_{0}$ is a redundant degree of freedom, which is reflected by the invariance under the following Weyl transformation which is a part of the super Weyl invariance in the full compensator formulation:

$$
C \rightarrow e^{-2 \tau} C, \quad g_{\mu \nu} \rightarrow e^{2(\tau+\bar{\tau})} g_{\mu \nu}, \quad \theta^{\alpha} \rightarrow e^{-\tau+2 \bar{\tau}} \theta^{\alpha},
$$

where $\tau$ is a complex constant. 
As stressed, $S_{\overline{D 3}}$ does not depend on the matter and gauge superfields confined in D7/D3. Then at the leading order in the supercovariant derivative expansion, $S_{\bar{D} 3}$ can be written as

$$
\begin{gathered}
S_{\bar{D} 3}=\int d^{4} x \sqrt{g^{C}} \int d^{4} \theta\left[-\frac{1}{2} e^{4 A_{m i n}} C^{2} C^{2 *} \theta^{2} \bar{\theta}^{2} P\left(\Phi^{m}, \Phi^{m *}\right)\right. \\
\left.+e^{3 A_{m i n}} C^{3} \overline{\theta^{2}} \Gamma\left(\Phi^{m}, \Phi^{m *}\right)+\text { h.c. }\right]
\end{gathered}
$$

where $P$ and $\Gamma$ are model-dependent functions of $\Phi^{m}$ and $\Phi^{m *}$, which are generically of order unity (in units with $M_{P l}=1$ ), and $e^{A_{\min }}$ is the (generically moduli-dependent) warp factor on $\bar{D} 3$. The $C$-dependence of $S_{\bar{D} 3}$ can be determined by requiring invariance of the $\bar{D} 3$ action under the Weyl transformation (28). Then the power of warp factor in each spurion operator is determined by the $C$-dependence since both $C_{0}$ and the warp factor correspond to the conformal mode of the $4 \mathrm{D}$ metric.

The $N=1$ SUSY appears to be explicitly broken in (29). In fact, the $N=1$ SUSY might be non-lineraly realized as suggested in [23] through a Goldstino fermion $\xi^{\alpha}$ living on the worldvolume of $\bar{D} 3$. In this case, the $\bar{D} 3$ action (29) can be extended to a manifestly supersymmetric form by replacing the $D$-spurion $\theta^{2} \bar{\theta}^{2}$ by a real superfield $\Lambda^{2} \bar{\Lambda}^{2}$, and the $F$-spurion by a chiral superfield $\left(\overline{\mathcal{D}}^{2}-8 \mathcal{R}\right) \Lambda^{2} \bar{\Lambda}^{2}$, where $\Lambda^{\alpha}$ is the Goldstino superfield defined in [24]:

$$
\Lambda^{\alpha}=\theta^{\alpha}+\frac{1}{M_{\bar{D} 3}^{2}} \xi^{\alpha}+\ldots
$$

for $M_{\bar{D} 3} \sim e^{A_{\min }} M_{s t}$, and $\left(\overline{\mathcal{D}}^{2}-8 \mathcal{R}\right)$ is the chiral projection operator of $4 \mathrm{D}$ SUGRA. Then the spurion operators of (29) can be obtained from the following form of super-Weyl invariant action involving the Goldstino superfield:

$$
S_{\bar{D} 3}=\int d^{4} x \sqrt{g^{C}} \int d^{4} \theta \tilde{C} \tilde{C}^{*} \mathcal{L}_{\bar{D} 3}\left(\frac{\tilde{C}^{1 / 2}}{\tilde{C}^{*}} \mathcal{D}_{\alpha}, \frac{\tilde{C}^{*}}{\tilde{C}^{1 / 2}} \Lambda^{\alpha}, \Phi^{m}\right),
$$

where $\tilde{C}=e^{A_{\min }} C$.

The coefficient function $e^{4 A_{\min } P}$ can be easily computed for the minimal KKLT model in which the only light modulus is the overall volume modulus $T$. In such model, the warp factor on $\bar{D} 3$ depends on $T$ as $e^{A_{\text {min }}}=$ $(\operatorname{Re}(T))^{1 / 4} e^{-2 \pi n / g_{s t} m}$ [4. Matching the $\bar{D} 3$ tension to (29) under the relation between the $4 \mathrm{D}$ Einstein frame metric $g_{\mu \nu}^{E}$ and the string frame metric 


$$
\begin{aligned}
& g_{s \mu \nu}=e^{2 A(y)}(\operatorname{Re}(T))^{-3 / 2} g_{\mu \nu}^{E}, \text { one finds } \\
& e^{4 A_{\min }} e^{2 K_{0} / 3} P=\frac{D}{\left(T+T^{*}\right)^{2}},
\end{aligned}
$$

where $D$ is a constant of order $M_{P l}^{4} e^{-8 \pi n / g_{s t} m}$.

\subsection{Soft terms in the presence of anti-D3 brane}

In the presence of $S_{\bar{D} 3}$ which breaks SUSY explicitly or realizes SUSY nonlinearly, the resulting soft terms are modified compared to the known results in standard 4D SUGRA. Solving the equations of motion for the auxiliary fields in $S=S_{N=1}+S_{\bar{D} 3}$, one easily finds

$$
\begin{aligned}
\frac{F^{C}}{C_{0}} & =\frac{1}{3} \partial_{I} K_{e f f} F^{I}+\frac{C_{0}^{* 2}}{C_{0}} e^{K_{e f f} / 3}\left(W_{e f f}+e^{3 A_{\min }} \Gamma\right)^{*} \\
F^{I} & =-\frac{C_{0}^{* 2}}{C_{0}} e^{K_{\text {eff }} / 3} K_{e f f}^{I \bar{J}}\left(D_{J}\left(W_{e f f}+e^{3 A_{\min }} \Gamma\right)\right)^{*}
\end{aligned}
$$

where $\left\{\Phi^{I}\right\}=\left\{\Phi^{m}, Q_{i}\right\}$, the Kähler covariant derivative $D_{I} X=\partial_{I} X+$ $\left(\partial_{I} K_{e f f}\right) X$, and one can choose $C_{0}=e^{K_{e f f} / 6}$ to arrive at the Einstein metric frame. It is then straightforward to find the following moduli potential and the soft parameters of the canonically normalized visible fields in the Einstein frame:

$$
\begin{gathered}
V_{0}=e^{K_{0}}\left[K_{0}^{m \bar{n}} D_{m}\left(W_{0}+e^{3 A_{\min }} \Gamma\right)\left(D_{n}\left(W_{0}+e^{3 A_{\min }} \Gamma\right)\right)^{*}\right. \\
\left.-3\left|W_{0}+e^{3 A_{\min }} \Gamma\right|^{2}\right]+e^{4 A_{\min }} e^{2 K_{0} / 3} P \\
\mathcal{L}_{\text {soft }}=-m_{i}^{2}\left|\tilde{Q}_{i}\right|^{2}-\left(\frac{1}{2} M_{a} \lambda^{a} \lambda^{a}+\frac{1}{6} A_{i j k} y_{i j k} \tilde{Q}_{i} \tilde{Q}_{j} \tilde{Q}_{k}+\text { h.c. }\right),
\end{gathered}
$$

where

$$
\begin{aligned}
M_{a} & =F^{m} \partial_{m} \ln \left(\operatorname{Re}\left(f_{a}\right)\right) \\
A_{i j k} & =-F^{m} \partial_{m} \ln \left(\frac{\lambda_{i j k}}{Y_{i} Y_{j} Y_{k}}\right) \\
& =-F^{m}\left(\partial_{m} K_{0}+\partial_{m} \ln \left(\frac{\lambda_{i j k}}{Z_{i} Z_{j} Z_{k}}\right)\right) \\
m_{i}^{2} & =\frac{2}{3} V_{0}-F^{m} F^{n *} \partial_{m} \partial_{\bar{n}} \ln \left(Y_{i}\right) \\
& =-\frac{1}{3} e^{4 A_{\min }} e^{2 K_{0} / 3} P+\left(V_{0}+m_{3 / 2}^{2}-F^{m} F^{n *} \partial_{m} \partial_{\bar{n}} \ln \left(Z_{i}\right)\right)
\end{aligned}
$$


for the canonically normalized Yukawa couplings

$$
y_{i j k}=\frac{\lambda_{i j k}}{\sqrt{Y_{i} Y_{j} Y_{k}}} .
$$

Here $Z_{i}=e^{K_{0} / 3} Y_{i}$ is the Kähler metric of $Q_{i}$, i.e.

$$
K_{\text {eff }}=K_{0}\left(\Phi^{m}, \Phi^{m *}\right)+Z_{i}\left(\Phi^{m}, \Phi^{m *}\right) Q_{i} Q_{i}^{*},
$$

and $m_{3 / 2}$ is the gravitino mass containing both the standard $N=1$ contribution from $W_{0}$ and the contribution from $\bar{D} 3$ :

$$
m_{3 / 2}=M_{P l} e^{K_{0} / 2}\left(W_{0}+e^{3 A_{\min }} \Gamma\right) .
$$

In the literature 10, $A_{i j k}$ and $m_{i}^{2}$ are normally expressed in terms of the moduli Kähler potential $K_{0}$ and the matter Kähler metric $Z_{i}$. Our results (35) show that it is more convenient to express those soft parameters in terms of the superspace wavefunction coefficient $Y_{i}=e^{-K_{0} / 3} Z_{i}$, particularly when the effects of the SUSY breaking $\bar{D} 3$ are included.

Obviously, when $P=\Gamma=0$, (33), (34) and (35) become the standard expressions of the SUSY breaking auxiliary components, the moduli potential and the soft terms in $N=1$ SUGRA. In KKLT models, in the absence of $\bar{D} 3, W_{0}$ leads to a SUSY AdS vacuum. Generically $P$ and $\Gamma$ are of order one for the moduli VEV of order unity, while $W_{0}$ is of order $m_{3 / 2} / M_{P l}$. Then, as we have noticed in sec. 2.2, in order for the AdS vacuum to be uplifted to a Minkowski vacuum by $\bar{D} 3$, one needs $e^{A_{\text {min }}} \sim \sqrt{m_{3 / 2} / M_{P l}}$. This implies that

$$
e^{3 A_{\min }} \Gamma \sim \sqrt{m_{3 / 2} / M_{P l}} W_{0},
$$

and thus the contribution to $m_{3 / 2}$ from $e^{3 A_{\min }} \Gamma$ is negligible compared to the contribution from $W_{0}$ induced by the flux and gaugino condensations. This means that $e^{3 A_{\min }} \Gamma$ can be safely ignored, so the effects of $\bar{D} 3$ on the low energy dynamics of the moduli and visible fields can be described well by a single coefficient function $P$. In such case, the SUSY breaking auxiliary components are well approximated by the standard $N=1$ expressions:

$$
\begin{aligned}
\frac{F^{C}}{C_{0}} & \simeq \frac{1}{3} \partial_{m} K_{0} F^{m}+M_{P l} e^{K_{0} / 2} W_{0}^{*}, \\
F^{m} & \simeq-M_{P l} e^{K_{0} / 2} K_{0}^{m \bar{n}}\left(D_{n} W_{0}\right)^{*} \\
m_{3 / 2} & \simeq M_{P l} e^{K_{0} / 2} W_{0},
\end{aligned}
$$


and the moduli scalar potential is approximately given by

$$
V_{0} \simeq M_{P l}^{4} e^{K_{0}}\left(K_{0}^{m \bar{n}} D_{m} W_{0}\left(D_{n} W_{0}\right)^{*}-3\left|W_{0}\right|^{2}\right)+V_{\text {lift }}
$$

where the uplifting potential from $\bar{D} 3$ is given by

$$
V_{\text {lift }}=e^{4 A_{\min }} e^{2 K_{0} / 3} P \text {. }
$$

Thus once $K_{0}$ and $W_{0}$ for the $N=1$ sector and $P$ for $\bar{D} 3$ are given, one can compute the SUSY breaking order parameters $F^{m}, F^{C}$ and $m_{3 / 2}$ using the above approximate results [2].

The soft parameters in (35) correspond to the moduli-mediated tree level contributions at the compactification scale, and do not include possible loop effects. As we will see, in KKLT-type models, $F^{C} / C_{0} \simeq m_{3 / 2}$ is bigger than the moduli-mediated soft masses typically by a factor $\sim 4 \pi^{2}$. With this little hierachy, the anomaly-mediated contributions from $F^{C} / C_{0}[9$ ] will be equally important as the moduli-mediated ones although they involve the loop suppression factor $1 / 8 \pi^{2}$. Then the dominant parts of the soft terms at energies just below the compactification scale can be obtained by simply adding the anomaly-mediated and also anomaly-moduli-mixed contributions to the moduli-mediated results (35). As is well known, such loop contributions are determined by the RG runnings of $Y_{i}$ and $f_{a}$, which are given by

$$
\begin{aligned}
\Delta \operatorname{Re}\left(f_{a}\right) & =-\frac{1}{16 \pi^{2}}\left(3 T_{a}(\operatorname{Adj})-\sum_{i} T_{a}\left(Q_{i}\right)\right) \ln \left(\frac{C C^{*}}{\mu^{2}}\right), \\
\Delta \ln \left(Y_{i}\right) & =-\frac{1}{32 \pi^{2}}\left(4 \sum_{a} \frac{C_{a}\left(Q_{i}\right)}{\operatorname{Re}\left(f_{a}\right)}-\sum_{j k} \frac{\left|\lambda_{i j k}\right|^{2}}{Y_{i} Y_{j} Y_{k}}\right) \ln \left(\frac{C C^{*}}{\mu^{2}}\right),
\end{aligned}
$$

where $T_{a}$ denotes the quadratic Casimir and $\mu$ is the renormalization point. Then replacing $f_{a}$ and $\ln \left(Y_{i}\right)$ in (35) by $f_{a}+\Delta f_{a}$ and $\ln \left(Y_{i}\right)+\Delta \ln \left(Y_{i}\right)$, and also $F^{m} \partial_{m}$ by $F^{m} \partial_{m}+F^{C} \partial_{C}$, we find the soft masses just below the compactification scale

$$
\begin{aligned}
M_{a} & =F^{m} \partial_{m} \ln \left(\operatorname{Re}\left(f_{a}\right)\right)+\frac{b_{a} g_{a}^{2}}{8 \pi^{2}} \frac{F^{C}}{C_{0}} \\
A_{i j k} & =-F^{m} \partial_{m} \ln \left(\frac{\lambda_{i j k}}{Y_{i} Y_{j} Y_{k}}\right)-\frac{\gamma_{i}+\gamma_{j}+\gamma_{k}}{16 \pi^{2}} \frac{F^{C}}{C_{0}}
\end{aligned}
$$




$$
\begin{aligned}
m_{i}^{2} & =\frac{2}{3} V_{0}-F^{m} F^{n *} \partial_{m} \partial_{\bar{n}} \ln \left(Y_{i}\right)-\frac{1}{32 \pi^{2}} \frac{d \gamma_{i}}{d \ln \mu}\left|\frac{F^{C}}{C_{0}}\right|^{2} \\
& +\frac{1}{16 \pi^{2}}\left(\gamma_{m}^{i} F^{m}\left(\frac{F^{C}}{C_{0}}\right)^{*}+\text { h.c }\right)
\end{aligned}
$$

where $b_{a}=-\frac{3}{2} T_{a}(\operatorname{Adj})+\frac{1}{2} \sum_{i} T_{a}\left(Q_{i}\right)$ are the one-loop beta function coefficients, $\frac{d g_{a}}{d \ln \mu}=\frac{b_{a}}{8 \pi^{2}} g_{a}^{3}, \gamma_{i}=2 \sum_{a} g_{a}^{2} C_{a}\left(Q_{i}\right)-\frac{1}{2} \sum_{j k}\left|y_{i j k}\right|^{2}$ are the anomalous dimension of $Q_{i}, \frac{1}{8 \pi^{2}} \gamma_{i}=\frac{d \ln Y_{i}}{d \ln \mu}, T_{a}$ and $C_{a}$ are group theory factors, $\operatorname{Tr}\left(T^{2}\right)=T_{a}\left(Q_{i}\right), \sum T^{2}=C_{a}\left(Q_{i}\right) I$, and finally $\gamma_{m}^{i}=\partial_{m} \gamma_{i}$ are given by

$$
\gamma_{m}^{i}=-\frac{1}{2} \sum_{j k}\left|y_{i j k}\right|^{2} \partial_{m} \ln \left(\frac{\lambda_{i j k}}{Y_{i} Y_{j} Y_{k}}\right)-2 \sum_{a} g_{a}^{2} C_{a}\left(Q_{i}\right) \partial_{m} \ln \left(\operatorname{Re}\left(f_{a}\right)\right) .
$$

If there is a Higgs bilinear term $H_{u} H_{d}$ either in the superpotential or in the Kähler potential, there will arise a soft $B$-term with $B \sim m_{3 / 2}$ at tree level. Since $m_{3 / 2} \sim 4 \pi^{2} M_{a}$ in our case, such $B$ would be too large to be phenomenologically acceptable. A simple way to avoid this difficulty is to assume that the Higgs $\mu$-term originates from a trilinear Yukawa term $\lambda N H_{u} H_{d}$ with an additional singlet field $N$, which would give $\mu=\lambda\langle N\rangle$ and $B=A_{N H_{u} H_{d}}$.

\section{Models}

In the previous section, we discussed the soft terms for a general form of $4 \mathrm{D}$ effective action $S_{4 D}=S_{N=1}+S_{\bar{D} 3}$ which describes the low energy limit of KKLT flux compactification. In this section, we consider some specific examples and compute the SUSY breaking order parameters, i.e. $F^{C}$ and $F^{\Phi}(\Phi=$ moduli), as well as the resulting soft terms of visible fields.

We will examine two simple classes of models, one in which $T$ is the only light modulus, and the other in which both $T$ and $S$ appear as light moduli. The first class of models represents a typical situation in Type IIB flux compactification with low energy SUSY, in which the dilaton $S$ and all complex structure moduli $Z^{\alpha}$ acquire superheavy masses $m_{S, Z} \sim 1 / M_{s t}^{2} R^{3} \sim 10^{16} \mathrm{GeV}$ from fluxes, while the Kähler modulus $T$ gets $m_{T} \sim m_{3 / 2} \ln \left(M_{P l} / m_{3 / 2}\right) \sim 10^{6}$ $\mathrm{GeV}$ from gaugino condensation. However, as was pointed out in [2], $S$ can be light also in some cases. For instance, if the NS fluxes are all vanishing, the flux-induced superpotential (9) would be independent of $S$ and $T$, and then only $Z^{\alpha}$ get superheavy masses of $\mathcal{O}\left(1 / M_{s t}^{2} R^{3}\right)$ from the RR fluxes. In 
this class of models there is no supersymmetric minimum before the uplifting. This runaway behavior can be cured if the superpotential acquires both $S$ and $T$ dependence from non-perturbative dynamics, for instance by gaugino condensations on $D 3$ and $D 7$ branes yielding $W_{n p}=C e^{-a T}+\Lambda e^{-b S}$. As for the uplifting potential $V_{\text {lift }}=e^{4 A_{\min }} e^{2 K_{0} / 3} P$ (in the Einstein frame) from $\bar{D} 3$, we will use $D / t^{n_{t}}$ for the first class of models, and $D / t^{n_{t}} s^{n_{s}}$ for the second class of models, where $T=t+i \tau, S=s+i \sigma$ and $D$ is a constant. As we will see, in all KKLT type models the anomaly mediation is generically equally or sometimes even more important than the moduli mediation.

\subsection{Models with light Kähler moduli}

The simplest KKLT-type model is the original one [1] described by

$$
\text { Model 1: } \begin{aligned}
K_{0} & =-3 \ln \left(T+T^{*}\right), \\
W_{0} & =w_{0}-C_{1} e^{-a T}, \\
V_{\text {lift }} & =D / t^{n_{t}}, \quad(T=t+i \tau) .
\end{aligned}
$$

For $K_{0}$ depending only on $t$, the overall phase of $W_{0}$ is irrelevant, and also the relative phase between $w_{0}$ and $C_{1}$ can be eliminated by shifting the axion field $\tau$ without any physical consequence. Then we can choose both $\omega_{0}$ and $C_{1}$ to be real and positive without loss of generality, in which case $\langle\tau\rangle=0$. Using the scalar potential given by (41) and also the expressions (40) for SUSY breaking order parameters, we find the model has a stable SUSY breaking Minkowski vacuum (when $D$ is tuned to make $\left\langle V_{0}\right\rangle=0$ ) with the following vevs and masses:

$$
\begin{aligned}
\frac{F^{C}}{C_{0}} \approx m_{3 / 2} & \approx M_{P l} \frac{w_{0}}{2 \sqrt{2} t^{3 / 2}} \\
\frac{F^{T}}{\left(T+T^{*}\right)} & \approx \frac{n_{t}}{2 a t} m_{3 / 2}, \\
m_{t} \approx m_{\tau} & \approx 2 a t m_{3 / 2}
\end{aligned}
$$

where

$$
a t \approx \ln \left(C_{1} / w_{0}\right) \sim 4 \pi^{2},
$$

and $n_{t}=2$ if $V_{\text {lift }}$ is from $\bar{D} 3$. Note that the parameter $C_{1}$ is of the order of 1 , while $w_{0}$ is of the order of $m_{3 / 2} / M_{P l}$, and then at is of order $4 \pi^{2}$ for 
$m_{3 / 2}$ close to the TeV scale. Such value of at gives rise to a little hierarchy between the moduli mass $m_{T}$, the gravitino mass and the gaugino/sfermion soft masses:

$$
m_{T} \sim 4 \pi^{2} m_{3 / 2} \sim\left(4 \pi^{2}\right)^{2} m_{\text {soft }} .
$$

A particularly interesting feature of the model is that

$$
\frac{F^{T}}{\left(T+T^{*}\right)} \sim \frac{1}{4 \pi^{2}} \frac{F^{C}}{C_{0}},
$$

and as a consequence the anomaly-mediation always gives a non-negligible contribution to soft masses.

In some cases, fluxes might preserve accidently a (discrete) $R$-symmetry, and thereby yield $w_{0}=0$. Still $T$ can be stabilized by introducing multiple gaugino condensations. Such case can be described by

$$
\text { Model 2: } \begin{aligned}
K_{0} & =-3 \ln \left(T+T^{*}\right), \\
W_{0} & =C_{1} e^{-a_{1} T}-C_{2} e^{-a_{2} T}, \\
V_{\text {lift }} & =D / t^{n_{t}} \quad(T=t+i \tau),
\end{aligned}
$$

where we can choose $C_{1}$ and $C_{2}$ to be real and positive without loss of generality and then set $\langle\tau\rangle=0$. In order to stabilize $t$ at a value yielding hierarchically small $m_{3 / 2} / M_{P l}$, one needs to tune $a_{1,2}$ as

$$
\left|a_{1}-a_{2}\right| \approx \frac{a_{1}+a_{2}}{\ln \left(M_{P l} / m_{3 / 2}\right)}
$$

as in the standard racetrack model 25$]$. We then find

$$
\begin{aligned}
\frac{F^{C}}{C_{0}} \approx m_{3 / 2} & \approx \frac{a_{2}-a_{1}}{2 \sqrt{2} a_{1} t^{\frac{3}{2}}} M_{P l} C_{2} e^{-a_{2} t}, \\
\frac{F_{T}}{\left(T+T^{*}\right)} & \approx \frac{3 n_{t}}{4 a_{1} t a_{2} t} m_{3 / 2}, \\
m_{t} \approx m_{\tau} & \approx \frac{4 a_{1} t a_{2} t}{3} m_{3 / 2},
\end{aligned}
$$

where

$$
a_{1} t \approx a_{2} t \approx \ln \left(M_{P l} / m_{3 / 2}\right) .
$$

The most important feature of this model is that $\frac{F_{T}}{\left(T+T^{*}\right)} \sim \frac{m_{3 / 2}}{(a t)^{2}}$ where at $\approx$ $4 \pi^{2}$, and as a consequence the soft terms are dominated by the anomalymediated contributions. 


\subsection{Models with light dilaton and Kähler moduli}

In this subsection, we examine some models in which both $T$ and $S$ are stabilized by a nonperturbative superpotential. Let us first consider the model given by

$$
\begin{aligned}
\text { Model 3: } K_{0} & =-\ln \left(S+S^{*}\right)-3 \ln \left(T+T^{*}\right), \\
W_{0} & =w_{0}-C_{1} e^{-a T}-\Lambda e^{-b S}, \\
V_{\text {lift }} & =D / t^{n_{t}} s^{n_{s}} \quad(T=t+i \tau, S=s+i \sigma),
\end{aligned}
$$

where $C_{1} e^{-a T}$ is induced by the gaugino condensation on $D 7$, while $\Lambda e^{-b S}$ is from the gaugino condensations on D3. Again we can choose $w_{0}, C_{1}$ and $\Lambda$ to be real and positive with appropriate shifts of $\tau$ and $\sigma$, and then $\langle\tau\rangle=\langle\sigma\rangle=0$. We then find

$$
\begin{aligned}
& a t \approx \ln \left(C_{1} / w_{0}\right) \approx \ln \left(M_{P l} / m_{3 / 2}\right), \\
& b s \approx \ln \left(\Lambda / w_{0}\right) \approx \ln \left(M_{P l} / m_{3 / 2}\right),
\end{aligned}
$$

and also

$$
\begin{aligned}
\frac{F^{C}}{C_{0}} \approx m_{3 / 2} & \approx \frac{w_{0}}{4 s^{1 / 2} t^{3 / 2}}, \\
\frac{F^{T}}{\left(T+T^{*}\right)} & \approx \frac{n_{t}}{2 a t} m_{3 / 2}, \\
\frac{F^{S}}{\left(S+S^{*}\right)} & \approx \frac{3 n_{s}}{2 b s} m_{3 / 2} .
\end{aligned}
$$

together with the dilaton/moduli masses

$$
m_{S, T} \approx m_{3 / 2} \ln \left(M_{P l} / m_{3 / 2}\right)
$$

Again the flux-induced $w_{0}$ in $W_{0}$ can be accidently vanishing, and $T$ and $S$ can be stabilized by having multi gaugino condensations either on $D 7$ or on $D 3$. In case that two gaugino condensations arise from $D 7$ branes, one would have

$$
\text { Model 4: } \begin{aligned}
K_{0} & =-3 \ln \left(T+T^{*}\right)-\ln \left(S+S^{*}\right) \\
W_{0} & =C_{1} e^{-a_{1} T}-C_{2} e^{-a_{2} T}-\Lambda e^{-b S} \\
V_{\text {lift }} & =D / t^{n_{t}} s^{n_{s}}
\end{aligned}
$$


For this model, we find

$$
a_{1} t \approx a_{2} t \approx b s \approx \ln \left(M_{P l} / m_{3 / 2}\right)
$$

and

$$
\begin{aligned}
\frac{F^{C}}{C_{0}} \approx m_{3 / 2} & \approx \frac{b s^{1 / 2}}{2 t^{3 / 2}} \frac{\Lambda e^{-b s}}{M_{P l}^{2}} \\
\frac{F^{T}}{\left(T+T^{*}\right)} & \approx \frac{3 n_{t}}{4 a_{1} t a_{2} t} m_{3 / 2}, \\
\frac{F^{S}}{\left(S+S^{*}\right)} & \approx \frac{3 n_{s}}{2 b s} m_{3 / 2} .
\end{aligned}
$$

The above results show that $\frac{F^{S}}{\left(S+S^{*}\right)} \sim \frac{F^{C}}{4 \pi^{2} C_{0}}$, thus $F^{S}$ and $F^{C}$ can give equally important contributions to soft terms. On the other hand, the $F$ component of $T$ stabilized by the racetrack superpotential is further suppressed as $\frac{F^{T}}{\left(T+T^{*}\right)} \sim \frac{F^{C}}{\left(4 \pi^{2}\right)^{2} C_{0}}$, thus gives negligible contribution to soft terms. Similarly, one can consider a model with

$$
W_{0}=\Lambda_{1} e^{-b_{1} S}-\Lambda_{2} e^{-b_{2} S}-C e^{-a T},
$$

and then one finds $\frac{F^{T}}{\left(T+T^{*}\right)} \sim \frac{F^{C}}{4 \pi^{2} C_{0}}$ and $\frac{F^{S}}{\left(S+S^{*}\right)} \sim \frac{F^{C}}{\left(4 \pi^{2}\right)^{2} C_{0}}$.

\subsection{Some phenomenological features}

Let us briefly discuss some phenomenological features of the soft terms in the models considered above. For the models of sec. 4.1, the matter Kähler metric and the holomorphic Yukawa couplings and gauge kinetic functions would be given by

$$
\begin{aligned}
\Delta K_{e f f} & =\frac{1}{\left(T+T^{*}\right)^{n_{i}}} Q_{i}^{*} Q_{i}, \\
\Delta W_{e f f} & =\frac{1}{6} \lambda_{i j k} Q_{i} Q_{j} Q_{k}, \\
f_{a} & =T^{l_{a}}
\end{aligned}
$$

where $\lambda_{i j k}$ are constants, $l_{a}=1, n_{i}=0$ and $l_{a}=0, n_{i}=1$ for gauge and matter fields living on D7 and D3, respectively. In the case when matter fields live on intersections of $D 7$ branes $n_{i}$ take a value between 0 and 1 [26]. 
Using the general result (43), the soft parameters of visible fields at energies just below the compactification scale are found to be [2]

$$
\begin{aligned}
M_{a}= & l_{a} \frac{F^{T}}{\left(T+T^{*}\right)}+\frac{b_{a} g_{a}^{2}}{2}\left(\frac{F^{C}}{4 \pi^{2} C_{0}}\right) \\
A_{i j k}= & \left(3-n_{i}-n_{j}-n_{k}\right) \frac{F^{T}}{\left(T+T^{*}\right)}-\frac{1}{4}\left(\gamma_{i}+\gamma_{j}+\gamma_{k}\right)\left(\frac{F^{C}}{4 \pi^{2} C_{0}}\right), \\
m_{i}^{2}= & \left(1-n_{i}\right)\left|\frac{F^{T}}{\left(T+T^{*}\right)}\right|^{2}-\frac{1}{32 \pi^{2}} \frac{d \gamma_{i}}{d \ln \mu}\left|\frac{F^{C}}{C_{0}}\right|^{2} \\
& +\left(\frac{1}{8} \sum_{j k}\left(3-n_{i}-n_{j}-n_{k}\right)\left|y_{i j k}\right|^{2}-\frac{1}{2} \sum_{a} l_{a} T_{a}\left(Q_{i}\right) g_{a}^{2}\right) \\
& \times\left(\frac{F^{T}}{\left(T+T^{*}\right)}\left(\frac{F^{* C}}{4 \pi^{2} C_{0}^{*}}\right)+\frac{F^{* T}}{\left(T+T^{*}\right)}\left(\frac{F^{C}}{4 \pi^{2} C_{0}}\right)\right) .
\end{aligned}
$$

For the models with the visible sector living on D3, we have $l_{a}=0$ and $n_{i}=1$, so $F_{T}$ does not contribute to the soft terms at tree-level. The no-scale structure is lifted by loop corrections and dependence of the soft terms on $F_{T}$ will appear at one-loop level. But, since $\frac{F^{T}}{\left(T+T^{*}\right)} \sim \frac{F^{C}}{4 \pi^{2} C_{0}}$ or even smaller, the soft terms are dominated by anomaly mediation. As is well known, pure anomaly mediation leads to negative slepton masses squared. In view of this point, the most attractive possibility would be the visible sector living on D7, in particular the model 1 in which the modulus mediation and the anomaly mediation give comparable contributions to the soft terms (for the model $2 F_{T}$ is too small to cure the negative slepton masses problem). It turns out 27] that the low energy phenomenology of such mixed modulusanomaly mediation is quite different from the pure anomaly mediation [9] and also from the pure modulus mediation [28]. In particular, since $F^{T}$ and $F^{C}$ have the same sign, the anomaly mediation cancels the RG evolution of the modulus-mediation, leading to a quite distinctive superparticle spectrum at low energy scale [27.

It should be stressed that the soft terms (63) have been derived within a framework satisfying two important conditions: (i) all relevant moduli are stabilized and (ii) the vacuum energy density is correctly tuned to be nearly zero. Unless the condition (i) is met, additional dynamics should be introduced to stabilize the unfixed moduli, and this new dynamics might change the predictions completely. The condition (ii) is also important for reliable 
computation of soft scalar masses since any additional source of vacuum energy density generically affects the soft scalar masses [29]. To our knowledge, this is the first example to compute soft terms in string theory framework satisfying these two conditions simultaneously.

To examine the structure of soft terms in models with light $T$ and $S$, one can consider the following forms of the matter Kähler metric and the gauge kinetic functions:

$$
\begin{aligned}
\Delta K_{e f f} & =\frac{1}{\left(S+S^{*}\right)^{k_{i}}\left(T+T^{*}\right)^{n_{i}}} Q_{i}^{*} Q_{i}, \\
\Delta W & =\frac{1}{6} \lambda_{i j k} Q_{i} Q_{j} Q_{k}, \\
f_{a} & =\kappa_{a} S+l_{a} T,
\end{aligned}
$$

where $\left(n_{i}, k_{i}\right)=(0,1)$ and $\left(n_{i}, k_{i}\right)=(1,0)$ for matter fields on $D 7$ and D3, respectively, and $\left(\kappa_{a}, l_{a}\right)=(0,1)$ and $\left(\kappa_{a}, l_{a}\right)=(1,0)$ for gauge fields living on $D 7$ and $D 3$, respectively. The resulting soft terms can be easily obtained from (43) as in 2. In this case a viable superparticle spectrum is obtained also for the visible sector living on D3.

Independently of the detailed low energy phenomenology [27], the soft parameters predicted by KKLT set-up have an attractive feature avoiding naturally the SUSY flavor and CP problems. The soft terms preserve the quark and lepton flavors if $n_{i}$, or $\left(n_{i}, k_{i}\right)$ for the models with light $T$ and $S$, are flavor-independent, which would arise automatically if the matter fields with common gauge charges live on the same $D$-brane worldvolume (or their intersection). They also preserve $\mathrm{CP}$ since the relative $\mathrm{CP}$ phase between $F^{T}$ and $F^{C} / C_{0}$ could be rotated away by the shift of the axion-like field $\tau$. Such dynamical relaxation of the potentially dangerous SUSY CP phase can be considered to be a consequence of an approximate nonlinear PQ symmetry $T \rightarrow T+i \alpha$ ( $\alpha=$ constant) which is broken by the stabilizing superpotential $W_{0}$ [30]. A similar relaxation of SUSY CP phases can be achieved in more general cases with multi-moduli $\Phi^{m}$ if the following conditions are met: (i) $W_{0}=\sum_{p} C_{p} \exp \left(-\sum_{m} a_{m}^{p} \Phi^{m}\right)$ and $f_{a}=\sum_{m} l_{a m} \Phi^{m}$ for real parameters $a_{m}^{p}$ and $l_{a m}$, (ii) the moduli Kähler potential, matter Kähler metric, and the uplifting potential depend only on $\Phi^{m}+\Phi^{* m}$, (iii) the number of independent terms in $W_{0}$ is limited as $N_{W_{0}} \leq N_{\Phi}+1$ where $N_{\Phi}$ is the number of involved moduli. In fact, these three conditions are satisfied in all models examined in this section. Finally we remark that the little mass hierarchy driven by the factor $\ln \left(M_{P l} / m_{3 / 2}\right) \sim 4 \pi^{2}$, i.e. $m_{T} \sim 4 \pi^{2} m_{3 / 2}$ and $m_{3 / 2} \sim 4 \pi^{2} m_{\text {soft }}$, allows 
the model to be free from the cosmological moduli problem and possibly also from the cosmological gravitino problem. It offers also an interesting scenario to produce a correct amount of neutralino dark matter as has been studied recently 31 .

\section{Conclusion}

A combination of fluxes and non-perturbative effects might allow for stabilization of all moduli of string theory. The KKLT set-up provides a specific suggestion how this can be achieved explicitely in the framework of the type IIB theory. It involves three steps: (i) a breakdown of supersymmetry through fluxes, (ii) a restoration of supersymmetry (in AdS) via nonperturbative effects, followed by (iii) again a breakdown of supersymmetry in the process of uplifting the vacuum energy to the desired value. The scheme requires a severe fine tuning of the fluxes to get a weak scale supersymmetry in addition to the other fine tuning for small cosmological constant. One might hope that future research could shed some light into the question of fine tuning and offer a more elegant description of the problem.

The study of the phenomenological properties of the scheme requires a careful analysis of the soft supersymmetry breaking terms. It is quite easy to compute the soft terms after the first step of the KKLT procedure, but this does not lead to meaningful results as in the second step supersymmetry is restored. From our analysis we can actually draw a useful lesson: it does not make sense to compute the soft terms in a scheme that has not yet stabilized all the moduli. The stabilization of the last modulus might (and usually does) change the results completely. So the first condition for a reliable computation of soft terms is to stabilize all the relevant moduli. Another important condition is that the framework should allow the vacuum energy density to be fine tuned to the desired small value since any additional source of vacuum energy density generically affects the soft scalar masses. To our knowledge, our analysis is the first attempt to compute soft terms in string theory framework satisfying these two conditions simultaneously.

The result of our analysis turns out to be rather simple and appealing. As we have shown, the KKLT scheme yields a unique and characteristic pattern of mass scales and soft terms. In case of a single light modulus (stabilized by nonperturbative effects) the result on mass scales is given in equation (20). We see that apart from the large hierarchy between the Planck scale and 
the weak scale, there appears a little hierarchy characterized by a numerical factor $\mathcal{O}\left(4 \pi^{2}\right)$ originating from $\ln \left(M_{P l} / m_{3 / 2}\right)$. One has $m_{T}=\mathcal{O}\left(4 \pi^{2} m_{3 / 2}\right)$ as well as $m_{3 / 2}=\mathcal{O}\left(4 \pi^{2} m_{\text {soft }}\right)$. The appearance of the soft terms is due to a specific mixed modulus-anomaly mediation, yielding the results of (43) and (63) which give a quite distinctive superparticle spectrum [27].

Independent of the details of the low energy phenomenology the soft parameters have the attractive feature of avoiding naturally the SUSY flavour and $\mathrm{CP}$ problems. In addition, the rather large value of the gravitino mass and the mass of the $T$-modulus could lead to interesting cosmological consequences [31. In the case of two light moduli the overall picture remains unchanged: the appearance of these little hierachies persists and the pattern of mass scales is quite similar.

\section{Acknowledgments}

This work was partially supported by the EU 6th Framework Program MRTN-CT-2004-503369 "Quest for Unification" and MRTN-CT-2004-005104 "ForcesUniverse" . K.C. is supported by Korean KRF PBRG 2002-070-C00022 and the Center for High Energy Physics of Kyungbook National University. A.F. was partially supported by the Polish KBN grant 2 P03B 12924 for years 2003-2005. M.O. was partially supported by the Polish KBN grant 2 P03B 00125 for years 2003-2005.

K.C. would like to thank the theory group of Bonn University for the hospitality during his visit. The stay of A.F. at DESY is possible due to Research Fellowship granted by Alexander von Humboldt Foundation.

\section{References}

[1] S. Kachru, R. Kallosh, A. Linde and S. P. Trivedi, Phys. Rev. D 68, 046005 (2003) arXiv:hep-th/0301240.

[2] K. Choi, A. Falkowski, H. P. Nilles, M. Olechowski and S. Pokorski, JHEP 0411, 076 (2004) arXiv:hep-th/0411066.

[3] J. Polchinski and A. Strominger, Phys. Lett. B 388 (1996) 736 arXiv:hep-th/9510227|. K. Becker and M. Becker, Nucl. Phys. B 477 (1996) 155 arXiv:hep-th/9605053. J. Michelson, Nucl. Phys. B 495 (1997) 127 arXiv:hep-th/9610151. K. Dasgupta, G. Rajesh and 
S. Sethi, JHEP 9908 (1999) 023 arXiv:hep-th/9908088. T. R. Taylor and C. Vafa, Phys. Lett. B 474 (2000) 130 arXiv:hep-th/9912152. P. Mayr, Nucl. Phys. B 593 (2001) 99 arXiv:hep-th/0003198. G. Curio, A. Klemm, D. Lust and S. Theisen, Nucl. Phys. B 609 (2001) 3 arXiv:hep-th/0012213. G. Curio, A. Krause and D. Lust, arXiv:hep-th/0502168

[4] S. B. Giddings, S. Kachru and J. Polchinski, Phys. Rev. D 66, 106006 (2002) arXiv:hep-th/0105097.

[5] J. P. Derendinger, L. E. Ibanez and H. P. Nilles, Phys. Lett. B 155, 65 (1985). M. Dine, R. Rohm, N. Seiberg and E. Witten, Phys. Lett. B 156, 55 (1985). J. P. Derendinger, L. E. Ibanez and H. P. Nilles, Nucl. Phys. B 267, 365 (1986). H. P. Nilles, arXiv:hep-th/0402022.

[6] I. R. Klebanov and M. J. Strassler, JHEP 0008 (2000) 052 arXiv:hep-th/0007191.

[7] S. Kachru, J. Pearson and H. Verlinde, JHEP 0206 (2002) 021 arXiv:hep-th/0112197.

[8] R. Brustein and S. P. de Alwis, Phys. Rev. D 69 (2004) 126006 arXiv:hep-th/0402088.

[9] L. Randall and R. Sundrum, Nucl. Phys. B 557, 79 (1999) arXiv:hep-th/9810155. G. F. Giudice, M. A. Luty, H. Murayama and R. Rattazzi, JHEP 9812, 027 (1998) arXiv:hep-ph/9810442. J. A. Bagger, T. Moroi and E. Poppitz, JHEP 0004, 009 (2000) arXiv:hep-th/9911029. P. Binetruy, M. K. Gaillard and B. D. Nelson, Nucl. Phys. B 604, 32 (2001) arXiv:hep-ph/0011081.

[10] S. K. Soni and H. A. Weldon, Phys. Lett. B 126, 215 (1983). V. S. Kaplunovsky and J. Louis, Phys. Lett. B 306, 269 (1993) arXiv:hep-th/9303040. A. Brignole, L. E. Ibanez and C. Munoz, Nucl. Phys. B 422, 125 (1994) [Erratum-ibid. B 436, 747 (1995)] arXiv:hep-ph/9308271.

[11] J. Polchinski, String theory. Vol. 2: Superstring theory and beyond

[12] P. G. Camara, L. E. Ibanez and A. M. Uranga, Nucl. Phys. B 689, 195 (2004) arXiv:hep-th/0311241. P. G. Camara, L. E. Ibanez and 
A. M. Uranga, arXiv:hep-th/0408036. M. Grana, T. W. Grimm, H. Jockers and J. Louis, Nucl. Phys. B 690, 21 (2004) arXiv:hep-th/0312232. T. W. Grimm and J. Louis, Nucl. Phys. B 699, 387 (2004) arXiv:hep-th/0403067|. H. Jockers and J. Louis, Nucl. Phys. B 705, 167 (2005) arXiv:hep-th/0409098. D. Lust, P. Mayr, R. Richter and S. Stieberger, Nucl. Phys. B 696, 205 (2004) arXiv:hep-th/0404134. D. Lust, S. Reffert and S. Stieberger, Nucl. Phys. B 706, 3 (2005) arXiv:hep-th/0406092. D. Lust, S. Reffert and S. Stieberger, arXiv:hep-th/0410074.

[13] K. Choi, Phys. Rev. D 56, 6588 (1997) arXiv:hep-th/9706171; Phys. Rev. D 62, 043509 (2000) arXiv:hep-ph/9902292.

[14] C. Munoz, arXiv:hep-th/9906152.

[15] S. Gukov, C. Vafa and E. Witten, Nucl. Phys. B 584, 69 (2000) [Erratum-ibid. B 608, 477 (2001)] arXiv:hep-th/9906070.

[16] S. Ashok and M. R. Douglas, JHEP 0401, 060 (2004) arXiv:hep-th/0307049 . F. Denef and M. R. Douglas, JHEP 0405, 072 (2004) arXiv:hep-th/0404116. A. Giryavets, S. Kachru and P. K. Tripathy, JHEP 0408, 002 (2004) arXiv:hep-th/0404243. L. Gorlich, S. Kachru, P. K. Tripathy and S. P. Trivedi, arXiv:hep-th/0407130.

[17] C. P. Burgess, L. E. Ibanez and F. Quevedo, Phys. Lett. B 447 (1999) 257 arXiv:hep-ph/9810535. K. Benakli, Phys. Rev. D 60 (1999) 104002 arXiv:hep-ph/9809582.

[18] V. Balasubramanian, P. Berglund, J. P. Conlon and F. Quevedo, arXiv:hep-th/0502058.

[19] O. DeWolfe and S. B. Giddings, Phys. Rev. D 67 (2003) 066008 arXiv:hep-th/0208123. J. F. G. Cascales, F. Saad and A. M. Uranga, arXiv:hep-th/0503079.

[20] C. P. Burgess, R. Kallosh and F. Quevedo, JHEP 0310, 056 (2003) arXiv:hep-th/0309187.

[21] P. Binetruy, G. Dvali, R. Kallosh and A. Van Proeyen, Class. Quant. Grav. 21, 3137 (2004) arXiv:hep-th/0402046. 
[22] H. Jockers and J. Louis, arXiv:hep-th/0502059.

[23] J. Bagger and A. Galperin, Phys. Rev. D 55 (1997) 1091 arXiv:hep-th/9608177|. M. Klein, Phys. Rev. D66, 055009 (2002) arXiv:hep-th/0205300; C. P. Burgess, E. Filotas, M. Klein and F. Quevedo, JHEP 0310, 041 (2003) arXiv:hep-th/0209190.

[24] S. Samuel and J. Wess, Nucl. Phys. B 221, 153 (1983).

[25] N. V. Krasnikov, Phys. Lett. B 193, 37 (1987). T. R. Taylor, Phys. Lett. B 252, 59 (1990). J. A. Casas, Z. Lalak, C. Munoz and G. G. Ross, Nucl. Phys. B 347 (1990) 243. B. de Carlos, J. A. Casas and C. Munoz, Nucl. Phys. B 399 (1993) 623 arXiv:hep-th/9204012.

[26] L. E. Ibanez, arXiv:hep-ph/0408064.

[27] K. Choi, K. S. Jeong and K. Okumura, in preparation.

[28] B. C. Allanach, A. Brignole and L. E. Ibanez, arXiv:hep-ph/0502151.

[29] K. Choi, J. E. Kim and H. P. Nilles, Phys. Rev. Lett. 73, 1758 (1994) arXiv:hep-ph/9404311.

[30] K. Choi, Phys. Rev. Lett. 72, 1592 (1994) arXiv:hep-ph/9311352.

[31] K. Kohri, M. Yamaguchi and J. Yokoyama, arXiv:hep-ph/0502211. 